\title{
A New Approach to Estimating the Fatigue Notch Factor of Ti-6Al-4V Components.
}

\author{
Gbadebo Owolabi*, Oluwamayowa Okeyoyin, Adewale Olasumboye, Horace Whitworth \\ Department of Mechanical Mechanical Engineering, Howard University \\ Washington, DC, USA, 20059 \\ *Corresponding author: Tel: 1-202-806-6594 Fax: 1-202-483-1396 \\ E-mail: gbadebo.owolabi@howard.edu
}

\begin{abstract}
Titanium alloy is used for airframe components and compressor blades application because of its high strength and fracture toughness at low temperatures and high strength and creep resistance at elevated temperature. This paper extends a recently developed probabilistic mesomechaniccs based model to notched titanium alloy components using simulation strategies that capture both the essence of notch root stress gradient and the complexity of realistic microstructures. The notch size effects and notch root and inelastic behavior are combined with probability distributions of microscale stress and small crack initiation to inform minimum life design methods. A new approach which can be applied using crystal plasticity finite element or closed form solution is also proposed as a more robust method for determining the fatigue notch factor than the existing classical methods. The fatigue notch factors predicted using the new probabilistic mesomechanics based model are in good agreements with experimental for notched titanium alloy specimens subjected to cyclic loads with various stress ratios.
\end{abstract}

Keywords: weakest link, fatigue notch factor, titanium alloy, closed-from solutions

\section{Introduction}

Titanium alloy Ti-6Al-4V offers a range of properties such as high strength and fracture toughness at low temperatures to high strength and creep resistance at elevated temperature. Thus, it is widely used in engineering applications from airframe components and fans to compressor blades of jet engines. Ingestion of debris into the engine of aircraft during takeoff and landing causes nicks and dents to form on the leading and trailing edge of turbine blades (Fig. 1). These dents and nicks can be treated as small notches with a notch root radius and notch depth and thus serve as stress raiser and favorable zones for crack initiation therefore reducing the fatigue strength of the material [1-3]. The fatigue strength reduction factor otherwise known as the fatigue notch factor, $\mathrm{k}_{\mathrm{f}}$, is used in the estimation of fatigue life and strength of notched structural components. Several models based on different assumptions have been developed for $\mathrm{k}_{\mathrm{f}}$ in the past. The Neuber [4], Kuhn et al. [5], Peterson [6], Heywood [7-8], Buch [9-10], and Siebel et al. [11] models are all based on average stress assumptions. One of the drawbacks of these models is that they do not incorporate explicit sensitivity to the combined effects of microstructure and strength of the notch root stress field gradient. Recent approaches have been developed to incorporate the stress gradient field at notches [12-13], but are deterministic and do not address the role of microstructure explicitly [14]. It is therefore very difficult to link the $\mathrm{k}_{\mathrm{f}}$ obtained using these methods to the realistic microstructure of the material such as grain size, grain orientation, multi-phases, and the notch root geometry. 


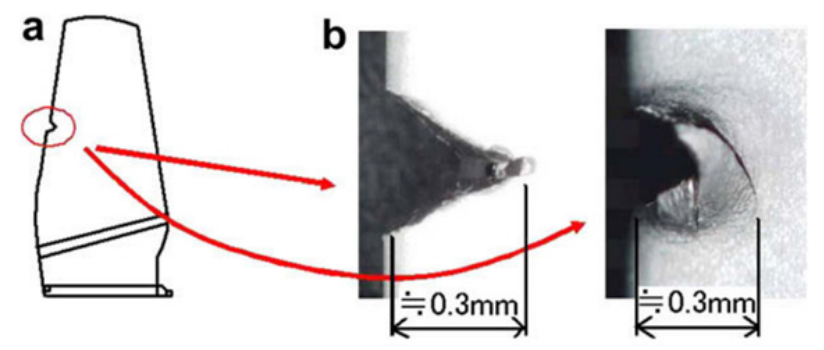

Fig.1. Image of foreign object damage; (a) Fan blade schematics (b) FOD damage example on edges of airfoil [2]

Owolabi et al. [14-15] recently developed probabilistic models based on the weakest link theory and extreme-value statistics where elements of crystal plasticity were combined with new probabilistic methods for notch sensitivity based on computed slip at the notch root within a well-defined fatigue damage process zone for homogenous oxygen free high conductivity copper. The purpose of this paper is to extend this model to heterogeneous dual-phase aeroengine materials such as the titanium alloy. The framework will incorporate information regarding not only the peak stress but also the stress gradient relative to microstructure length scale. This approach can reduce the amount of testing required to make design decisions on material or component reliability by systematically estimating the scatter of fatigue life associated with microstructure variations through the use of simulations.

\section{Material and Crystal Plasticity Models}

Titanium is widely known for its good resistance to corrosion and high strength to weight ratio. When alloyed with other metals and heat treated, it can achieve a wide range of attractive properties both at low and high temperatures. Titanium alloys exhibit high strength and creep resistance at high temperatures while at low temperatures, they exhibit high strength and fracture toughness. At room temperature, an unalloyed titanium has an hcp crystal structure but with increase in temperature to the neighborhood of $882^{\circ} \mathrm{C}$, it undergoes a change in phase from the hcp $\alpha$ phase to a bcc $\beta$ phase. It is possible to produce a completely $\alpha$ - phase, $\beta$ - phase, or a mixture of $\alpha$ and $\beta$ phases by varying the alloying elements. Different microstructures can be realized for the same alloy by varying the processing parameters and heat treatment. Titanium alloy is dual phase and it has the inherent problem of varying individual phase properties due to different amount of alloying elements. The hcp structure of titanium, unlike for fcc structured materials, has several planes which are favorable for occurrence of slip or twinning. In most hcp materials, basal (0001) and prismatic $\{1010\}$ have been identified as the primary slip planes with a closed packed direction $\langle 1120\rangle$ for the slip vector. Titanium has been identified to exhibit flow stress versus temperature anomaly as well as orientation dependent yielding according to the work of Naka et al. [16].

The crystal plasticity model used in this work followed the existing work of Mayeur and McDowell [17], Zhang et al. [18] and Bridier et al. [19] for Ti-6Al-4V. The duplex Ti-6Al-4V is a dual-phase alloy consisting of an hep structured matrix primary a-phase and secondary lamellar $\alpha+\beta$ domains with alternating layers of secondary $\alpha$ laths and bec structured residual $\beta$ laths. The crystal plasticity model accounts for the distinct three-dimensional slip geometry for each phase, anisotropic and length scale dependent slip system strengths, the non-planar dislocation core structure of prismatic screw dislocations in the primary a-phase, and crystallographic texture [18]. The relationship between the slip system shearing rate and the resolved shear stress of the $\alpha$ slip system is described by the power law flow rule given as: 
$\dot{\gamma}^{\alpha}=\dot{\gamma}_{0}\left\langle\frac{\left|\tau^{\alpha}-\chi^{\alpha}\right|-\kappa^{\alpha}}{D^{\alpha}}\right\rangle^{m} \operatorname{sgn}\left(\tau^{\alpha}-\chi^{\alpha}\right)$

where, $\gamma_{o}$ is the reference shearing rate, $\mathrm{m}$ is the flow exponent which controls the rate sensitivity of flow, $\tau^{\alpha}$ is the resolved shear stress, $\chi^{\alpha}$ is the back stress, $\kappa^{\alpha}$ is the length scale-dependent threshold stress and $D^{\alpha}$ is the drag stress. The drag stress is taken as a non-evolving constant, i.e. $\dot{D}^{\alpha}=0$, while the back stress evolves according to an Armstrong-Frederick direct hardening/dynamic recovery type of equation [19], i.e.

$\dot{\chi}^{\alpha}=h \dot{\gamma}^{\alpha}-h_{D} \chi^{\alpha}\left|\dot{\gamma}^{\alpha}\right|$

with $\chi^{\alpha}(0)=0$. The threshold stress is expressed as [19]:

$\kappa^{\alpha}=\frac{\kappa_{y}}{\sqrt{d^{\alpha}}}+\kappa_{s}^{\alpha}$

In equation (3), the first term uses a Hall-Petch-type formulation with $d^{\alpha}$ as the microstructural dimension of the free slip length and $\kappa_{\mathrm{y}}$ as the Hall-Petch constant. The second term corresponds to the softening parameter that addresses the breakdown of short range order due to the dislocation glide commonly observed in a-Ti-6Al-4V at room temperature where planar slip of screw dislocations promotes a nearly elastic-perfectly plastic behavior [19]. The threshold stress evolution law based on softening of all slip systems is of the form [19]:

$\dot{\kappa}_{s}^{\alpha}=-\mu \kappa_{s}^{\alpha}\left|\dot{\gamma}^{\alpha}\right|$

where $\mu$ is a constant.

It is noted that the cyclic behavior of titanium dual-phase titanium alloy depends significantly on the activity of twinning and the interaction of dislocations with twins [20]. The deformation microstructure at room temperature depends, however, on the applied strain amplitude. Thus, for the purpose of this study only slip is assumed to be the main mechanism of plastic deformation. This assumption is reasonable since under low strain amplitudes as in the case of high cycle fatigue considered here, the influence of twinning is minimal; thus, the primary deformation mechanism in Ti-6Al-4V (like all $\alpha / \beta$ Ti-Al alloys) is the prismatic glide of $\langle a\rangle$-type screw dislocations [17,20]. Moreover, for Ti-6Al-4V alloy, William et al [21] have shown that the occurrence of twinning decreases with increasing aluminium content and in titanium alloy with $6 \%$ or higher atomic wt. \% aluminum, twinning is hardly observed [20]. Thus, with the role of twinning being negligible in many $\alpha / \beta$ alloys, the importance of $\mathbf{c}+\mathbf{a}$ slip is enhanced since it is the sole mechanism responsible for accommodating deformation along the c-axis [20].

It is very difficult to explicitly model the $\alpha+\beta$ colonies containing the secondary $\alpha$ and $\beta$ phases arranged in lamellar structure since the thicknesses of the laths are much smaller structures when compared to the microstructural statistical volume element of the titanium alloy using a finite element mesh that must include a large number of grains [19]. To model the $\alpha+\beta$ 
colonies, we followed the approach detailed in references [17-20] where the slip geometry of lamellar colonies is obtained by homogenizing the lamellar structure systems in an equivalent grain-scale representation of the $\alpha+\beta$ colonies based on the burgers orientation relation (BOR) given by $(0001)_{\alpha} / /\{101\}_{\beta}$ and $(11 \overline{2} 0)_{\alpha} / /\{111\}_{\beta}$ as shown in Fig 2. There are 24 possible slip systems in the lamellar region: $3\langle 11 \overline{2} 0\rangle(0001)$ basal, $3\langle 11 \overline{2} 0\rangle\{10 \overline{1} 0\}$ prismatic, $6\langle 11 \overline{2} 0\rangle\{10 \overline{1} 1\}$ first order pyramidal and $12\langle 111\rangle\{110\}$ bcc slip systems which are transformed into the hexagonal coordinate system according to the BOR [18]. The readers are referred to references [17-19] for more details on this modeling approach.

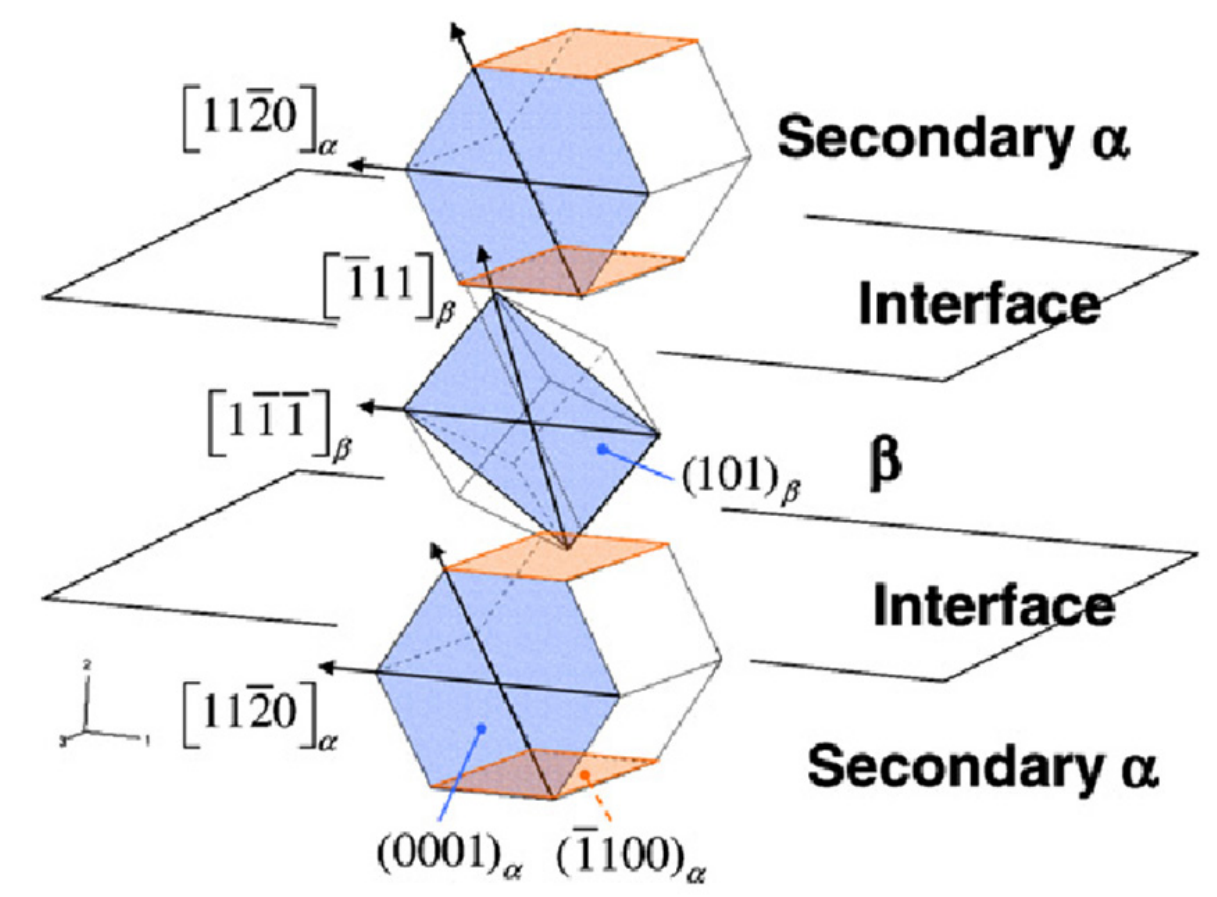

Fig. 2. Lamellar orientation relationships [18].

\section{Three Dimensional Finite Element Implementation Procedure for Notched Titanium Alloy Component}

The crystal plasticity models presented is Section 2 is coded into ABAQUS 2006 UMAT. The elastic constants $\left(c_{i j}\right)$ are obtained from the work of Simmons et al. [22] on single crystals and have been adjusted based on the measurements by Larson et al. [23] on strongly textured Ti$6 \mathrm{Al}-4 \mathrm{~V}$ alloy. Some of the material parameters for the textured Ti-6Al-4V in the crystal plasticity codes are obtained from Bridier et al. [19] and are as presented in Table 1. 
Table 1: Ti-6Al-4V crystal plasticity model parameters

\begin{tabular}{lll}
\hline Elastic stiffness & $C_{11}$ & $162,400 \mathrm{MPa}$ \\
& $C_{12}$ & $92,000 \mathrm{MPa}$ \\
& $C_{13}$ & $69,000 \mathrm{MPa}$ \\
& $C_{33}$ & $180,700 \mathrm{MPa}$ \\
Flow rule & $C_{44}$ & $49,700 \mathrm{MPa}$ \\
& $\gamma_{0}$ & $0.001 \mathrm{~s}^{-1}$ \\
Back stress & $M$ & 15 \\
& $\chi^{\alpha}(0)$ & 0 \\
Threshold stress & $h$ & 40,000 \\
& $h_{D}$ & 8000 \\
$\kappa_{y}$ & $17.3 \mathrm{MPa} \mathrm{mm} \mathrm{m}^{-0.5}$ \\
& $\kappa_{s}^{\alpha}(0)$ & $150 \mathrm{MPa}$ \\
& $\mu$ & 50 \\
\hline
\end{tabular}

The notched geometry modeled in this work is a v-notched cylindrical component schematically represented as shown in Fig. 3. Finite element simulations were performed on three different geometries and meshed using 3D stress four-node linear tetrahedron element type (C3D4) to estimate the stress distribution in the notch region of the specimens. The dimensions of the specimens used and the different test cases are as given in Table 2 obtained from [24].

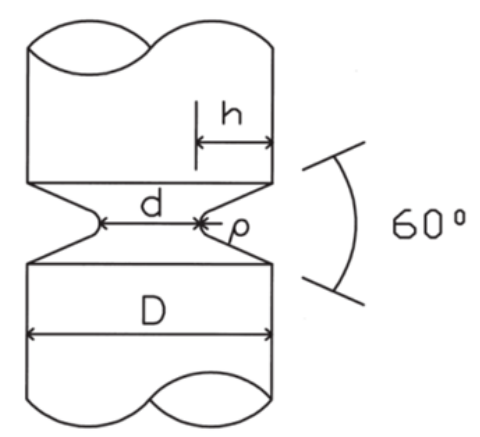

Fig. 3: Gage section of the cylindrical specimen with a circumferential V-notch.

Table 2: Notch-root test cases

\begin{tabular}{cccccc}
\hline $\begin{array}{c}\text { Test } \\
\text { Case }\end{array}$ & $\mathrm{K}_{\mathrm{t}}$ & $\begin{array}{c}\text { Notch radius, } \\
\boldsymbol{\rho}(\mathrm{mm})\end{array}$ & $\begin{array}{c}\text { Notch depth, h } \\
(\mathrm{mm})\end{array}$ & R-ratio & $\begin{array}{c}\text { Average alternating HCF } \\
\text { strength at } 10^{6} \text { cycles (MPa) }\end{array}$ \\
\hline 1 & 2.78 & 0.330 & 0.729 & -1 & 173.6 \\
2 & 2.78 & 0.330 & 0.729 & 0.1 & 158.9 \\
3 & 2.78 & 0.330 & 0.729 & 0.5 & 104.6 \\
4 & 2.78 & 0.203 & 0.254 & 0.1 & 167.2 \\
5 & 2.78 & 0.203 & 0.254 & 0.5 & 105.2 \\
6 & 2.78 & 0.127 & 0.127 & 0.1 & 144.7 \\
7 & 2.78 & 0.127 & 0.127 & 0.5 & 111.0 \\
\hline
\end{tabular}


To reduce computational time, two different simplifications were adopted. The notched specimen geometries are decomposed into three different regions: an outermost region, far from the notch root, where isotropic linear elasticity is used; an intermediate transition region where macroscopic $\mathrm{J}_{2}$ cyclic plasticity theory is used; and finally the notch root region where crystal plasticity theory is used [14]. It is important to note that the element size at the outermost and J2 plastcity region was chosen to coincide with the average grain size of Ti-6Al-4V which is $45 \mu \mathrm{m}$. Mesh refinement study was however initially conducted to determine the maximum element size required for convergence for the crystal plasticity region. Four different mesh seed sizes were used for meshing each grain in the crystal plasticity region; $45 \mu \mathrm{m}$ (i.e. the grain size, $\mathrm{d}_{g r}$ ), $22.5 \mu \mathrm{m}\left(\mathrm{d}_{g r} / 2\right), 15 \mu \mathrm{m}\left(\mathrm{d}_{g r} / 3\right)$ and $11.25 \mu \mathrm{m}\left(\mathrm{d}_{g r} / 4\right)$ with the notch root radius of $330 \mu \mathrm{m}$. These represent respectively $1,2,3$, and 4 elements through each grain thickness. For all simulations, remote applied stress amplitude of $173.6 \mathrm{MPa}$ was imposed with a stress ratio of $\mathrm{R}_{\varepsilon}=-1$ For the purpose of this study, a percentage error of $1 \%$ or less was taken as a convergence criterion. The simulation results show significant difference between the extreme-values of the nonlocal maximum stress for the coarse mesh seed sizes. A mesh seed size of 3 elements per grain thickness produced an error of $0.30 \%$ when compared to the 4 element per grain thickness. Therefore a minimum of 3 elements per grain thickness, which corresponds to a maximum of $15.0 \mu \mathrm{m}$ mesh seed size was chosen for this study. To maintain consistency and for the purpose of comparison, the same number of elements per grain thickness was used for all notch root. Secondly, one quarter of the cylindrical notched specimen was modeled because of the symmetry in loading and geometry of the specimen. Symmetry boundary conditions are applied at the planes of symmetry. The bottom of the notched specimen is encastre while symmetry boundary conditions are applied to the two planes of symmetry making up a total of 3 boundary conditions. The notched specimens were tested at three different load ratios; $\mathrm{R}=0.1,0.5$, and -1 for ten different randomly generated grain distribution around the notch root.The average alternating HCF strength at $10^{6}$ cycles as determined by Naik et al [24] and shown in Table 2 is applied to the top of the specimen.

\section{Probabilistic Model for Fatigue Notch Factor}

The probabilistic framework used to obtain the fatigue notch factor and the associated probability of fatigue at the scale of grain follows the framework presented in [14-15] with a few modifications based on the physical mechanisms of fatigue crack formation and growth for heterogeneous aero-engine materials. This is important as fatigue is manifested by extremal microstructure attributes that promote slip intensification and hence does not conform to homogenization. Following the framework presented in [14-15], the probability of survival of a notched component having a fatigue damage process zone of volume $\mathrm{V}_{\mathrm{d}}$, divided into small volume elements, $\mathrm{dV}$ with probability of survival of a sufficiently small volume element is given as:

$$
P_{s}=\exp \left(\int_{V_{d}}-\frac{1}{V_{0}}\left(1+\xi\left(\frac{\sigma-\sigma_{t h}}{\sigma_{0}}\right)\right)^{-1 / \xi} d V\right)
$$

where $\xi, \sigma_{\text {th }}$ and $\sigma_{0}$ represent the Weibull shape, location and scale parameters respectively. The shape parameter, $\xi$, is a measure of the scatter in the stress, $\sigma$ distribution among grains within the damage process zone $\mathrm{V}_{\mathrm{d}}$ at the notch root; $\sigma_{\text {th }}$ is the threshold stress below which no

microdamage will occur at a given number of cycles in the high cycle fatigue (HCF) regime, and $\mathrm{V}_{0}$ is a reference volume.

The probabilistic framework presented in [14-15] focuses on the formation of a fatigue crack on the scale of a single grain, and will therefore be applicable to the high cycle fatigue 
(HCF) and very high cycle fatigue (VHCF) regimes for which total life is strongly correlated to crack formation at this scale. It is also assumed that slip drives crack nucleation and growth of small cracks to the scale of grains; a crack may form in any of the grains within the damage process zone, $\mathrm{V}_{\mathrm{d}}$, defined as the smallest volume corresponding to a locus of points enclosing grains with stresses, $\sigma$ above some threshold, $\sigma_{\text {th }}$. The damage process zone $V_{d}$ in the notch root region is composed of a number of grains, each with volume $\mathrm{dVi}$, considered as infinitesimal relative to $\mathrm{V}_{\mathrm{d}}$. Eq. (5) as:

The cumulative probability of HCF failure of the notched component is obtained from

$P_{f}=1-\exp \left(\int_{V_{d}}-\frac{1}{V_{0}}\left(1+\xi\left(\frac{\sigma-\sigma_{t h}}{\sigma_{0}}\right)\right)^{-1 / \xi} d V\right)$

Using Eq. (6) and following the probabilistic framework presented in Owolabi et al. [15], a new relation is obtained for the microstructure-dependent fatigue notch factor of the form:

$$
k_{f}=\frac{\sigma_{\max , s}}{\sigma_{\max , n}}=\psi\left(\frac{V_{n}}{V_{s}}\right)^{\xi}
$$

where

$$
\psi=\left[\frac{1}{V_{d}} \int_{V}\left(\frac{\sigma_{o}+\xi\left(\sigma-\sigma_{\text {th }}\right)}{\sigma_{\text {net }}}\right)^{-1 / \xi} \mathrm{dV}\right]^{\xi}
$$

where $\psi$ can be regarded as the microscopic stress concentration factor and $\sigma_{\text {net }}$ is the net section stress of the notched component computed as the average of the stress in the net section region outside the damage process zone. Vn and Vs are the volume of the notch and the smooth specimens respectively. The yield stress $\sigma_{\text {th }}$ for the titanium alloy is $930 \mathrm{MPa}$ [24], $\xi$ is 0.13 , while the scale parameter $\sigma_{0}$ is $3205.03 \mathrm{MPa}$. Both $\xi$ and $\sigma_{\mathrm{o}}$ were determined using the modified moment method in Owolabi et al [15]. From the geometry of the smooth specimen, the reference volume $\mathrm{V}_{0}$ is calculated to be $16.96 \mathrm{~mm}^{3}$.

\section{Closed Form Solution for Fatigue Notch Factor}

To resolve inelastic deformation at the scale of microstructure to facilitate next generation microstructure-sensitive notch root analyses inherently requires mesh refinement to the scale of microstructure, which is often several orders of magnitude finer than the scale of the component. Moreover, the kind of constitutive equations that must be used are often of advanced form, for example, discrete dislocation mechanics, requiring rather sophisticated and time-consuming 
computational strategies to perform concurrent analyses at the component and notch root microstructure scales. Accordingly, direct application of multiscale finite element analysis is simply too computationally time consuming for practical microstructure-sensitive fatigue damage assessment of notched components under multiaxial loads. Thus, for practical engineering application, a more simplified and approximate model for fatigue notch factor is presented here based on closed form solution for stress distribution at the notch developed by Glinka using the Creager-Paris solutions of the stress field ahead of a crack. For a notch component with notch root radius $\rho$ and stress concentration factor, $k_{t}$, the axial stress distribution along the notch root is given as:

$$
\sigma=\frac{1}{2}\left(\left(\frac{\rho}{x+\rho / 2}\right)^{1 / 2}+\frac{1}{2}\left(\frac{\rho}{x+\rho / 2}\right)^{3 / 2}\right) \sigma_{\max , n}=\frac{1}{2}\left(\left(\frac{\rho}{x+\rho / 2}\right)^{1 / 2}+\frac{1}{2}\left(\frac{\rho}{x+\rho / 2}\right)^{3 / 2}\right) k_{t} S
$$

If $\sigma_{\mathrm{ac}}$ is the value of the stress at the critical length where $\mathrm{x}=\mathrm{a}_{\mathrm{c}}$, Eq (9) can be re-written as:

$$
\sigma_{a_{c}}=\frac{1}{2}\left(\left(\frac{\rho}{a_{c}+\rho / 2}\right)^{1 / 2}+\frac{1}{2}\left(\frac{\rho}{a_{c}+\rho / 2}\right)^{3 / 2}\right) k_{t} S
$$

The ratio $o \phi \sigma_{\mathrm{ac}}$ to the nominal stress $\mathrm{S}$ in $\mathrm{Eq}(10)$ is the equivalent of the $\psi$ in $\mathrm{Eq}$ (8), i.e.,

$$
k_{t}=\psi=\frac{\sigma_{a_{c}}}{S}=\frac{1}{2}\left(\left(\frac{\rho}{a_{c}+\rho / 2}\right)^{1 / 2}+\frac{1}{2}\left(\frac{\rho}{a_{c}+\rho / 2}\right)^{3 / 2}\right)
$$

Using Eq (11) in Eq (7) will allow the determination of an expression for the fatigue notch factor of the form:

$$
k_{f}=\left(\frac{1}{2}\left(\left(\frac{\rho}{a_{\mathrm{c}}+\rho / 2}\right)^{1 / 2}+\frac{1}{2}\left(\frac{\rho}{a_{\mathrm{c}}+\rho / 2}\right)^{3 / 2}\right)\right)\left(\frac{V_{n}}{V_{s}}\right)^{\xi}
$$

The above closed-form equation for $\mathrm{k}_{\mathrm{f}}$ is thus derived using the probabilistic framework based on the weakest link and the Glinka's closed form solution based on the notch root stress distribution 


\section{Results and Discussion}

Seven different test cases in Table 2 were simulated in ABAQUS with ten different grain orientation distributions for each of the test case. The stress distributions for some of the test cases simulated are shown in Figure 4 for the stress in the axial direction. The stress distribution within the fatigue damage process zone $V_{d}$ was used in Eq. (6) to determine the probability of failure for each test case. Also, the fatigue notch factor is computed for each test case using Eq. (7) and the average values is compared with the experimental values from literature. The fatigue damage process zone used in this work followed the one defined in Owolabi et al [15]. However, the fatigue damage process zone used here is based on the distribution of the stress within the notch root region. The statistical distribution of stress, $\sigma$, around the notch root is used as a new criterion in defining the fatigue damage process zone. It is based on the assumption that the fatigue damage process zone is a region around the notches having grains with $\sigma$ values equal to or greater than a specified threshold value, $\sigma_{\text {th }}$. Here, the yield stress of the material is used as the threshold value.

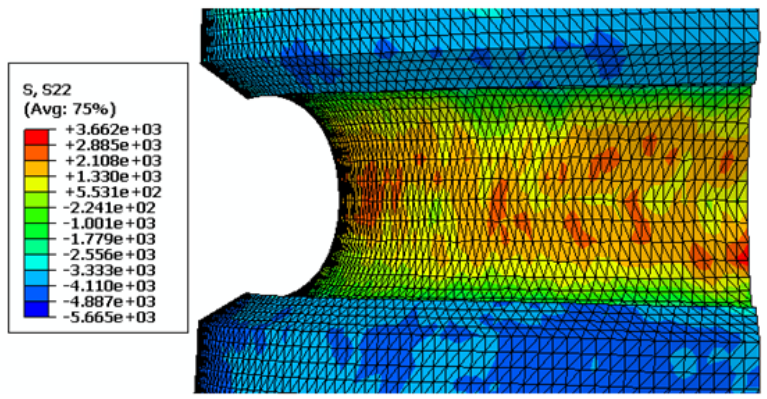

(a)

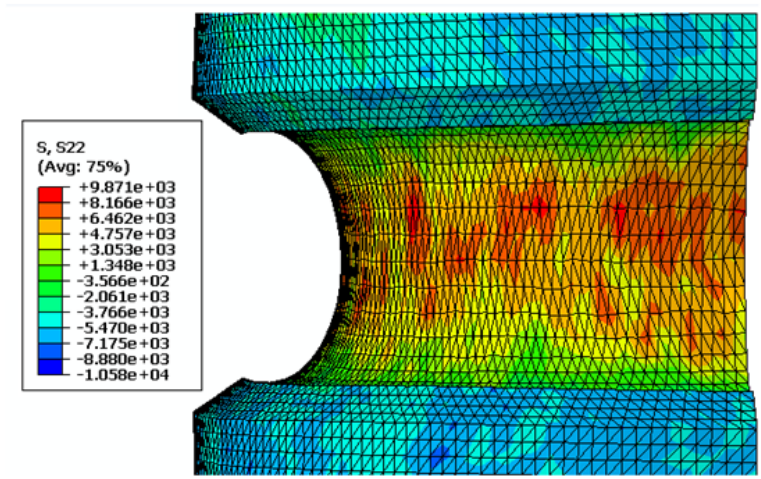

(c)

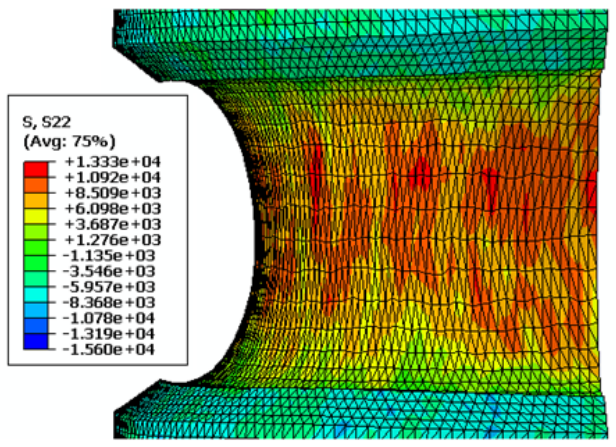

(b)

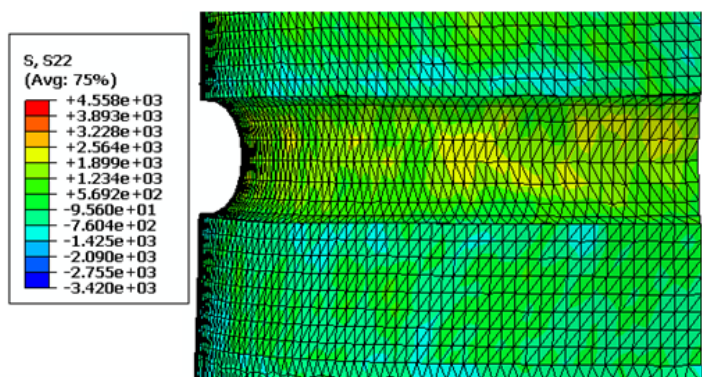

(d)

Fig. 4. Stress distribution for notched titanium alloy: (a) $\rho=0.33, R=-1$ and remotely applied stress of 173.6 MPa (b) $\rho=0.33, \mathrm{R}=0.1$ and remotely applied stress of $158.9 \mathrm{MPa}$ (c) $\rho$ $=0.33, \mathrm{R}=0.5$ and remotely applied stress of $104.6 \mathrm{MPa}$ (d) $\rho=0.203, \mathrm{R}=0.1$ and remotely applied stress of 167.2 $\mathrm{MPa}$. (All results in $\mathrm{MPa}$ ) 
Figure 5 shows probability of failure plotted against the notch root radii for the ten realizations with different random grain orientation distributions at s stress ratio of 0.1 . Figure 5 shows that the probability of failure increases with increasing notch root radius. However, an exception is noted for titanium alloy specimen with $0.127 \mathrm{~mm}$ notch root radius where some of the probabilities of failure for some of the grain orientations are higher than for the remaining notch root radii $(0.203 \mathrm{~mm}$ and $0.33 \mathrm{~mm})$. This is an indication that grain orientation around the notch root region also plays a significant role in determining the occurrence of fatigue failure in the notched titanium alloy specimen. This microstructure-sensitive probabilistic approach thus enables us to gain direct insight into the controlling physical phenomena at the microstructure scale and offers improved identification of cause-effect relations for the material for scatter in fatigue life data due to microstructure variability and notch/component size effects.

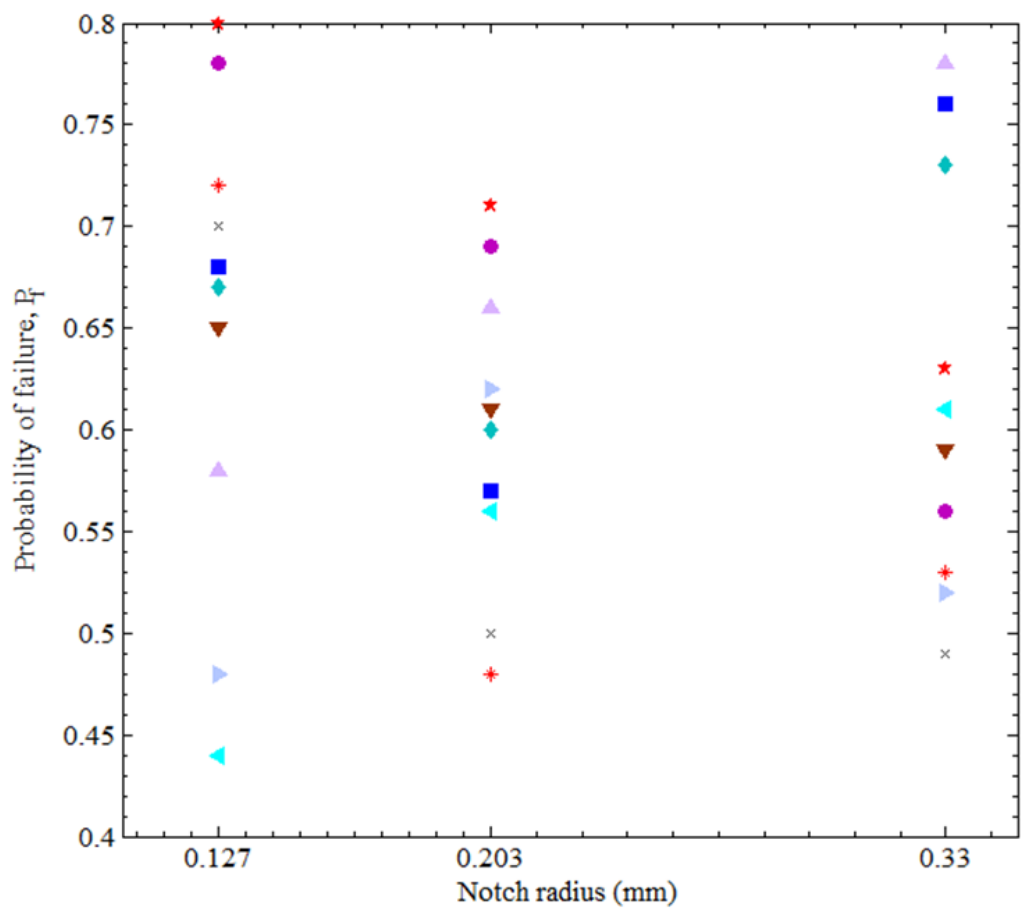

Fig. 5. Probability of failure vs. notch radius for notched titanium alloy at applied remote stress of $158.9 \mathrm{MPa}$ and stress ratio $\mathrm{R}=0.1$ for ten realizations with different random grain orientation distributions.

The average of the fatigue notch factor determined using the probabilistic framework for the ten different random grain orientation is compared to the experimentally obtained values from Table 1 of Naik et al. [24], the closed-from solution developed and other existing conventional method such as Neuber as shown in Figure 6 and Table 3. It can be noted from Table 3 that the percentage variance for the Weibull-based fatigue notch factor and the closedform solution is lower compared to the Neuber's predictions. Thus, the developed probabilistic model and the close form solution are more accurate in predicting fatigue notch factor of notched titanium alloy compared to Neuber method. In view of the association of the calculated fatigue notch factor with the formation of a crack on the order of grain size, and in view of the dominance of the first major barrier (e.g., grain boundary) in arresting growth of microstructurally small cracks, we may regard the fatigue notch factor obtained via the probabilistic approach in the long life limit as relating closely to fatigue limit behavior for 
notched components. In the low cycle and transition fatigue regimes for which the cyclic plastic strain amplitude at the notch root substantially exceeds the elastic strain amplitude, it is likely that this particular basis for the fatigue notch factor will have less utility than a formulation that considers small crack propagation through a network of grains at the notch root [14].

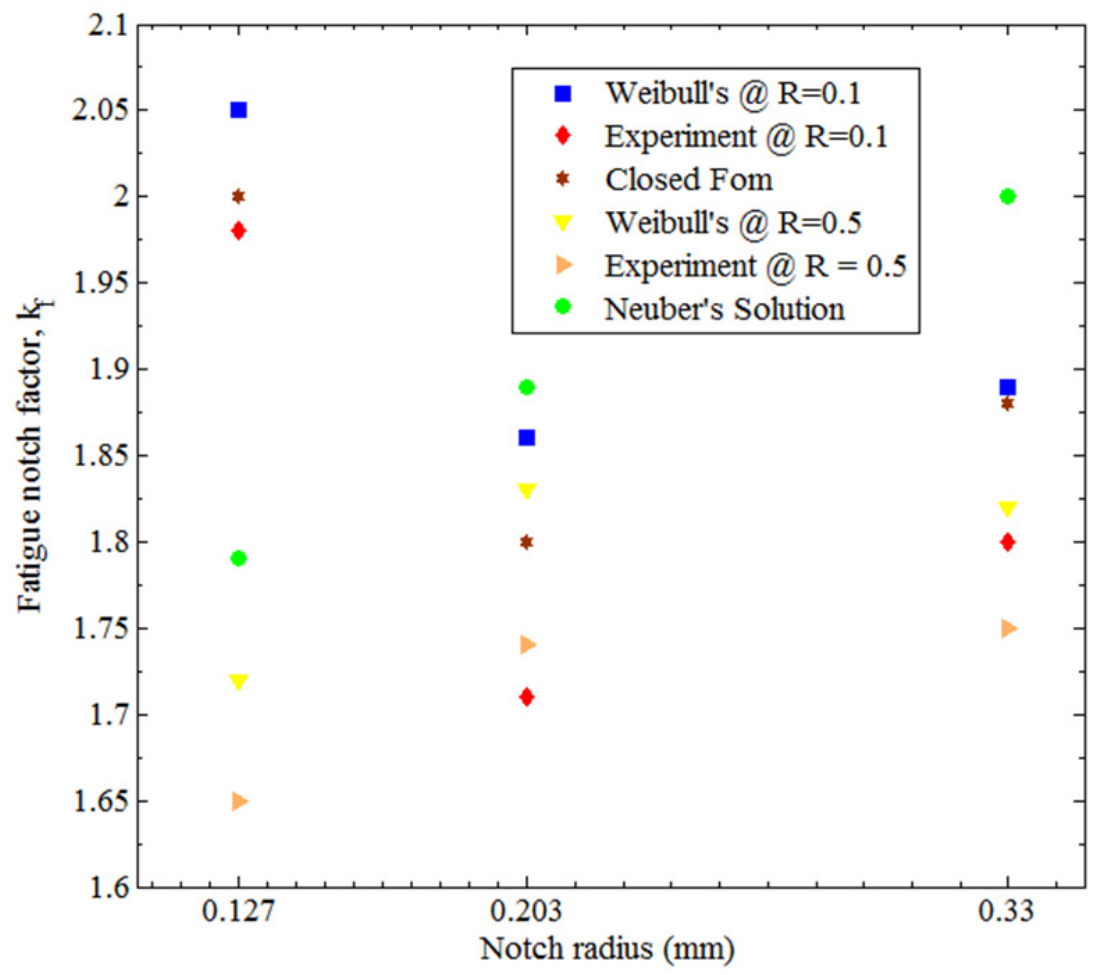

Fig. 6. Fatigue notch factor as a function notch root radius and load ratio compared to experimental values.

Table 3: Fatigue notch factor \% variance from experimental value for Weibull, close form solution and Neuber methods

\begin{tabular}{rcccccccccc}
\hline Radius & \multicolumn{2}{c}{ Experiment $\mathrm{k}_{\mathrm{f}}$} & \multicolumn{2}{c}{ Weibull@ $\mathrm{R}=0.1$} & \multicolumn{2}{c}{ Weibull@ $\mathrm{R}=0.5$} & \multicolumn{2}{c}{ Neuber } & \multicolumn{2}{c}{$\begin{array}{c}\text { Closed Form } \\
\text { Sol. }\end{array}$} \\
& $\mathrm{R}=0.1$ & $\mathrm{R}=0.5$ & $\mathrm{~K}_{\mathrm{f}}$ & $\%$ Var & $\mathrm{k}_{\mathrm{f}}$ & $\%$ Var & $\mathrm{k}_{\mathrm{f}}$ & $\%$ Var & $\mathrm{k}_{\mathrm{f}}$ & $\%$ Var \\
\hline 0.127 & 1.98 & 1.65 & 2.05 & -3.54 & 1.72 & -4.24 & 1.79 & 9.60 & 2.00 & -1.01 \\
0.203 & 1.71 & 1.74 & 1.86 & -8.77 & 1.83 & -5.17 & 1.89 & -10.53 & 1.80 & -5.26 \\
0.33 & 1.80 & 1.75 & 1.89 & -5.00 & 1.82 & -4.00 & 2.00 & -11.11 & 1.88 & -4.44 \\
\hline
\end{tabular}

\section{Conclusions}

A recently developed probabilistic model for microstructure- sensitive fatigue notch factor was extended to titanium alloy components. The model makes use of the stress distribution result obtain from finite element crystal plasticity simulation of notched components with various notch geometries subjected to cyclic loads with various stress ratios. The result shows that the 
probability of failure and the fatigue notch factor increase with increasing notch root radius. Also it is noted that the grain orientation of the material plays an important role in determining or predicting the fatigue strength of the material; the probability of failure varies at the same notch root radius for different grain orientation.

\section{Acknowledgements}

The authors of this paper express their profound gratitude to the Department of Defense for the financial support provided through contract \# W911NF-11-1-041 (Dr. Asher Rubinstein, Program Manager, Army Research Office and Dr. D. Stargel, Program Manager, Air Force Office of Scientific Research.)

\section{References}

1. Haritos GK, Nicholas T, \& Lanning DB. Notch size effects in HCF behavior of Ti-6Al4V. Int. J. Fatigue 1999; 21: 643-652.

2. Yamashita Y, Ueda Y, Kuroki H, Shinozaki M. Fatigue life prediction of small notched Ti-6Al-4V specimens using critical distance. Engineering Fracture Mechanics, 2010; 77: 1439-1453.

3. Carter TJ.Common failures in gas turbine blades. Engineering Failure Analysis 2005; 12 : 237-247

4. Neuber HJ. Theory of stress concentration for shear-strained prismatical bodies with arbitrary nonlinear stress-strain law. J. Appl. Mech 1961; 28(4): 544-550.

5. Kuhn P, Hardraht HF. An engineering method for estimating the notch-size effect in fatigue tests on steel. In NACA TN2805 Langley Aeronautical Laboratory Washington 1952.

6. Peterson RE. Notch sensitivity. In Metal Fatigue, G. Sines and J. Waisman, Eds., New York, McGraw-Hill 1959:293-306.

7. Heywood RB. Stress concentration factors. Engineering 1955; 179: 146.

8. Heywood RE. Design against fatigue. Chapman \& Hall London 1962.

9. Buch A. Analytical approach to size and notch -size effect in fatigue of aircraft materials specimens. Material Science and Engineering 1974; 15: 75-85.

10. Buch A. Fatigue strength calculation. Switzerland: Trans Tech Publications 1998.

11. Siebel E, Stieler M. Significance of dissimilar stress distributions for cycling loading. VDI- Zeitschrift 1955; 97 (5): 121-126.

12. Adib-Ramezani H. Jeong J. Advanced volumetric method for fatigue life prediction using stress gradient effects at notch root. Computational Material Science 2007; 39: 649-663.

13. Ren W. Nicholas T. Notch size effects on high cycle fatigue limit stress of udimet 720. Material Science and Engineering 2003; A357: 141-152.

14. Owolabi GM, Prasannavenkatesan R, McDowell DL. Probabilistic framework for a microstructure-sensitive fatigue notch factor. International Journal of Fatigue 2010; 32: 1378-1388. 
15. Owolabi G, Egboiyi B, Shi L, Withworth H. Microstructure-dependent fatigue damage process zone and notch Sensitivity Index," International Journal of Fracture 2011; 170: 159-173.

16. Naka S, Lasalmonie A, Costa P, Kubin LP. The low-temperature plastic deformation of alpha- titanium and the core structure of a-type screw dislocations. Phil. Mag 1988; 57: 717-740.

17. Mayeur J.R. McDowell DL. A three-dimensional crystal plasticity model of duplex Ti6Al-4V. Int Journal of Plasticity 2007; 23: 1457-1485.

18. Zhang M. Zhang J. McDowell DL. Microstructure-based crystal plasticity modeling of cyclic deformation of Ti-6Al-4V. Int Journal of Plasticity 2007; 23: 1328-1348.

19. Bridier F. McDowell D.L., Villechaise P., Mendez J. Crystal plasticity modeling of slip activity in Ti-6Al-4V under high cycle fatigue loading. Int J Plasticity 2009; 25:10661082.

20. Mayeur J. Three dimensional modeling of titanium-aluminum alloys with application to attachment fatigue. MS thesis, Georgia Institute of Technology, 2004.

21. Williams, JC., Baggerly, RG., Paton, NE. Deformation behavior of HCP Ti-Al alloy single crystals, Met. Mat. Trans, 2002, 33: 837-850.

22. Simmons G. Wang H. Single crystal elastic constants and calculated aggregated properties. A Handbook, Cambridge M.A : MIT Press 1971.

23. Larson F, Zarkades A. Properties of textured titanium alloys. Batelle memorial institute, Columbus OH, 1974.

24. Naik RA, Lanning DB, Nicholas T, Kallmeyer AR. A critical plane gradient approach for the prediction of notched HCF life. Int J Fatigue 2005; 27:481-492. 